\title{
Three-dimensional strain analysis of single-lap bolted joints in thick composites using fibre-optic gauges and the finite-element method
}

\author{
G Restivo $^{1}$, G Marannano ${ }^{2 *}$, and G A Isaicu ${ }^{1}$ \\ ${ }^{1}$ Mechanical Engineering Department, Michigan State University, East Lansing, Michigan, USA \\ ${ }^{2}$ Dipartimento di Meccanica, Università degli Studi di Palermo, Palermo, Italy
}

The manuscript was received on 15 September 2009 and was accepted after revision for publication on 12 February 2010.

DOI: $10.1243 / 03093247 J S A 599$

\begin{abstract}
Bolted joints involving composite plates used to be almost entirely dedicated to aerospace applications. As the need for energy conservation has increased, the field of composite bolted joints has found new applications in ground armoured vehicles. Thick panels able to withstand large in-plane and impact loads are critical. The present investigation evaluates the interior strain field, through the thickness, of a composite plate connected to an aluminium panel with a single-lap bolted joint. The area of interest is the bearing plane region close to the hole because of the presence of stress concentrations that heavily modify the stress field. Experimental data for the bolted joint were recorded by fibre-optic strain gauges that were embedded in the bearing plane of the composite plate. Numerical analyses were performed using ANSYS as a pre-processor and LS-DYNA as a solver. The overall goal was to evaluate the magnitude of contact strains around the hole and through the thickness of the composite. These values were analysed and compared with the finite-element method results: the finite-element analysis correlated reasonably well with the experiments. An investigation of error causes was also carried out, in particular to evaluate the influence of incorrect gauge positioning and the effect of friction coefficients. General design considerations were finally provided, based on the complete three-dimensional finite-element analysis.
\end{abstract}

Keywords: bolted joint, composite materials, fibre-optic strain gauge (FOSG), FEM, ANSYS, LS-DYNA

\section{INTRODUCTION}

Fastening composite structures introduces yet another problem in the search for a complete understanding of their behaviour. The simplest methods to fasten composites are bolting, riveting, and bonding. Bonding and riveting do not allow for easy removal or replacement of damaged parts and inspection under limited space conditions. Bolting composites, however, proves to be a very inexpensive and efficient solution. Parts can be easily removed and replaced, and structural integrity can be evaluated with ease. To take advantage of fibre-reinforced

*Corresponding author: Dipartimento di Meccanica, Università degli Studi di Palermo, Viale delle Scienze, Palermo, Italy. email: marannano@dima.unipa.it polymer (FRP) composite structures, complete analyses of stress and strain distributions in the composite structure are needed. The influence of stress concentration factors such as the presence of the bolt hole, bolt hole to plate edge distance, hole diameter, and bolt-to-hole clearance have to be fully understood.

In an extended experimental study on damage development in mechanically fastened laminate composites, Ireman et al. [1] used graphite/epoxy laminates in an attempt to characterize damage development close to the bolt hole. The study used both single and multi-bolt configurations of singlelap joints in conjunction with a special fixture to eliminate the bending moment caused by the eccentric load path (secondary bending). Both countersunk and protruding head bolts were used. 
Damage development was assessed using resistance strain gauges, acoustic emission, X-ray, and by microscopic examinations. The specimens were loaded a number of times (six or seven) and monitored by acoustic emission. When acoustic emission showed that some damage occurred to the specimen, the loading was stopped and the specimen was examined by X-ray and microscope. The study concluded that the specimens tend to experience the whole palette of FRP failure modes before total failure is reached. As the load increases the specimens show matrix cracking, fibre failure, delamination, and kinking followed by total failure.

Hou and Liu [2] studied the effects of size and thickness on the composite joints. Glass/epoxy specimens with $[0,90]$ stacking sequence of 9,17 , and 34 plies were employed in a double-lap pin joint configuration. Regarding the effect of joint length on the composite joint, the study concluded that as the ratio between the length from the hole to the clamped end over the width passed 4 , the joint strength remains fairly constant. Investigations of three-dimensional (3D) size effects revealed that the ratio of thickness over the hole diameter was found to be a critical value for the composite joint. The effect of pin-hole clearance values over the joint was clear, with the joint strength decreasing as the clearance increased. As the loading rate increased, an increase in the joint strength was observed. The study also concluded that as the FRP plate thickness increased, a decrease in strength was observed.

Tong [3] studied the bearing failure of composite bolted joints with non-uniform bolt-to-washer clearance. The study focused on the relative positions of the bolt and washer on the bearing failure of the bolted joint. Composite plates were made of carbon/ epoxy using a quasi-isotropic stacking sequence. The specimens were set to have eight plies of plain carbon weave. Steel bolts, nuts, and washers were used for the joining of the composite panel to two steel plates in a double lap configuration. A piezoelectric load cell transducer was used to measure clamping loads. The present authors observed good agreement for the case when the washer is offset in the loading direction and poor agreement for the case when the washer is offset opposite to the loading direction. Possible causes that may influence the agreement between experimental and analytical results are listed. The study concludes that the extreme positions of the washer to the bolt shank slightly affects the initial failure load but has no significant influence on the ultimate failure load.
Previous finite-element method (FEM) models built to simulate bolted joints were mainly twodimensional (2D). One of first studies concerned with 3D stress analysis of a composite laminate bolted joint was developed by Chen et al. [4]. The study reports on a general method of evaluating contact stresses generated by the bolt-hole interaction using an incremental variational principle formulation and the transformation matrix derived from $3 \mathrm{D}$ contact kinematic conditions. The numerical results obtained were compared with experimental results from a double-lap bolted joint. The composite plates used in the experiment were thin graphite/epoxy with $[ \pm 45 /$ 0/90]S quasi-isotropic lay-up and thick glass/epoxy of various stacking sequences. The effects of friction, clearance, bolt elasticity, stacking sequence, and clamping on the contact tractions around the bolted joint were included in the analysis. Clamping force was modelled as pressure and it was determined analytically. Good agreement between the numerical results and the referenced experimental results was reported.

Ireman [5] did a 3D stress analysis of bolted single-lap composite joints. A 3D finite-element model of the composite bolted joint was developed to determine non-uniform stress distributions through the thickness of composite laminates in the proximity of the bolt hole. The finite-element models were created using IDEAS and solved using Abaqus, a commercially available finite-element software. Added supports were used to eliminate secondary bending owing to offset boundary conditions. One coefficient of friction was used for all contact surfaces owing to software limitations. Numerical results were compared against strain gauge measurements.

Most of the past studies concentrated on 2D finiteelement numerical models, and just a few studies approached the 3D finite-element numerical models. From the 3D finite-element models one can observe that most of them are concerned with either double-lap bolted joints or single-lap bolted joints modified such that the bending caused by the eccentric loading path is not included in the analysis. Furthermore, previous experimental studies contained data from resistance strain gauges applied on the surface of the composite plate. This approach does not allow measurements very close to the bolt hole, or on the bearing plane of the specimen. One study included resistance strain gauges inside an isotropic specimen with fairly good results [6]. Using resistance strain gauges inside a laminate composite plate would not be desirable 
because it could alter the mechanical properties of the composite plate in a significant manner.

The goal of the present research was to conduct a combined numerical-experimental study of a singlelap bolted joint involving a thick composite laminate and an aluminium plate. The bolted joint was modelled numerically using finite-element analysis (Ansys-LS-DYNA), and it included all the fastening parts (washers, nut, and bolt) as separate entities (Fig. 1).

The numerical results were then compared with experimental data coming from two fibre-optic strain gauges embedded in the composite plate. The ability to obtain data from within the specimen provides a deeper understanding of the strain field and a more meaningful validation of the numerical results.

\section{EXPERIMENTAL STUDY}

Two glass/epoxy specimens were manufactured for this experimental study. The first one was used as a tensile specimen to determine the elastic properties of the final laminate. The second one was connected to an aluminium plate (2024-T4) via a single-lap bolted joint. Material properties for the glass fibre (weave style 3733) and epoxy resin (Epoxies, Etc... 20-3302) used for both specimens are listed in Table 1. In order to obtain the desired thickness of $12.5 \mathrm{~mm}, 60$ plies of bidirectional 0/90 E-glass weave

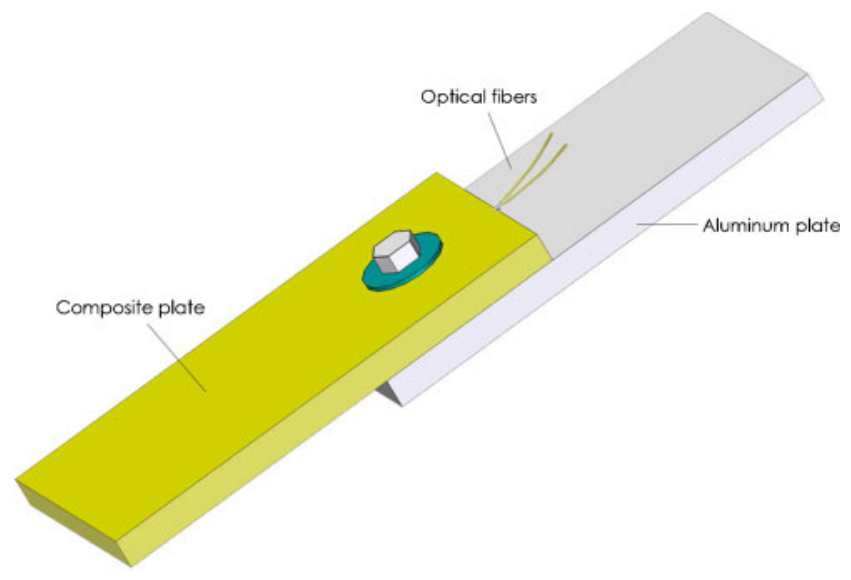

Fig. 1 Single-lap bolted joint

Table 1 Isotropic material properties

\begin{tabular}{lcl}
\hline & $\begin{array}{l}\text { Young's modulus, } E \\
(\mathrm{MPa})\end{array}$ & $\begin{array}{l}\text { Poisson's } \\
\text { ratio, } v\end{array}$ \\
\hline E-glass fibre & 72300 & 0.22 \\
Epoxy resin & 3500 & 0.37 \\
Aluminium 2024-T4 & 69600 & 0.34 \\
\hline
\end{tabular}

were used during the lay-up phase, which was performed by hand. The final fibre volume fraction was calculated to be around 38 per cent.

\subsection{Material properties validation}

For the tensile test, a combination of MicroMeasurements EA-13-120LZ-120 0-90 resistance strain gauge rosettes and fibre-optic strain gauges was used. By using both methods, the conventional and well-known resistance strain gauges, and the new generation of sensors - the fibre-optic strain sensors $[\mathbf{7}, \mathbf{8}]-$ a validation of the fibre-optic sensors was also possible.

All fibre-optic strain gauges used were Fabry-Perot type gauges having a $2 \mathrm{~mm}$ gauge length, as manufactured by FISO Technologies. The outer diameter of the strain gauge was $230 \mu \mathrm{m}$, and the range of measurement was $\pm 2500 \mu \varepsilon$. Because of the small size and weight of the gauges, they could be embedded in the specimen at the time of fabrication and they did not significantly affect the material properties of the thick composite laminate plate. The data stream coming from the sensors could be used at any given time for validation purposes, as well as for health monitoring of the composite joint during its service life.

Fibre-optic strain gauges were embedded in the composite specimens at the time of fabrication. In order to maintain the position of the sensor during lay-up and curing processes, the fibre-optic sensors were attached to the fibreglass cloth. Each sensor was positioned and held in place while epoxy resin was applied with a small brush. A sample attachment of a fibre-optic sensor to the E-glass weave can be seen in Fig. 2.

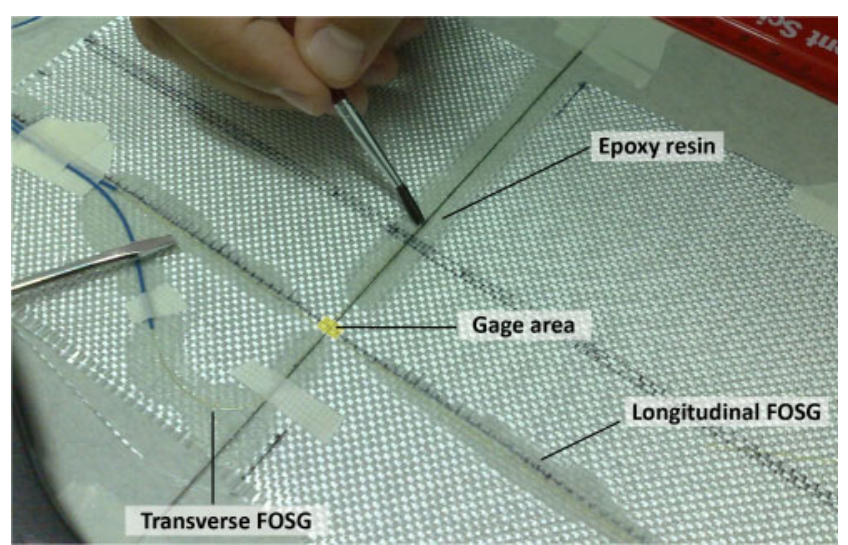

Fig. 2 Fibre-optic strain gauge (FOSG) glued to the Eglass weave 
The RSGs were connected to Measurements Group P-3500 strain indicator set-ups in half-bridge configuration with temperature compensation gauges mounted on an unloaded specimen. The FISO fibreoptic strain gauges were connected to an eightchannel FISO UMI-8 signal conditioner that provided a data stream to a portable laptop computer [9].

The tensile test specimen, as shown in Fig. 3, was $250 \mathrm{~mm}$ long, $50 \mathrm{~mm}$ wide, and $12.7 \mathrm{~mm}$ thick. It was mounted on a swivel fixture to eliminate bending, and loaded in tension from 0 to $900 \mathrm{~N}$. Tensile test data (Fig. 4) were interpreted in order to obtain the elastic orthotropic properties that would be used in the FEM simulations. These values are shown in Table 2.

The advantages of using orthotropic material properties for the finite-element numerical models are not insignificant. Calculation time is greatly reduced because of the use of lower-order elements having a lower number of degrees of freedom
(DOFs). Convergence problems are also avoided owing to the simplified model.

\subsection{Single-lap bolted joint}

The goal here is to obtain experimental strain values through the thickness and around the hole for the composite plate. These results will be used to validate a 3D finite-element model. In turn, the finite-element model, validated by experimental data, delivers the locations of the maximum contact stress and of the possible failure of the composite plate. The composite specimen dimensions and aluminium plate dimensions are shown in Fig. 5 .

Two fibre-optic sensors were used to measure strains inside the composite plate, in the bearing plane. Figure 6 shows the position of each fibreoptic sensor. The distance between the two sensors in the bearing plane is equal to $2.54 \mathrm{~mm}$.

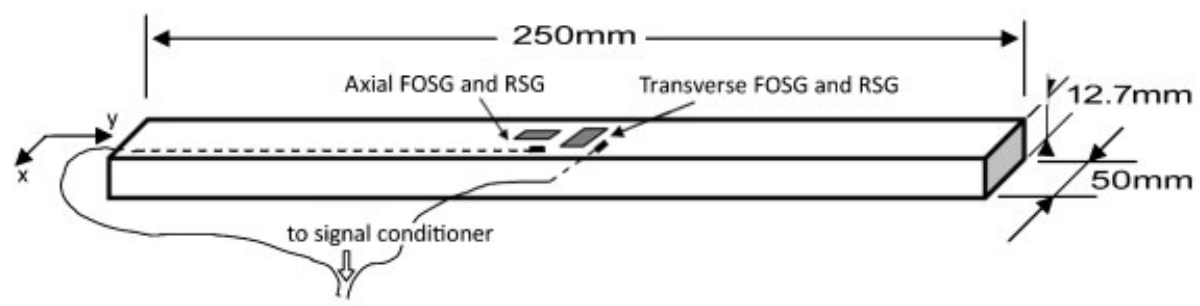

Fig. 3 Tensile test specimen and strain gauge layout

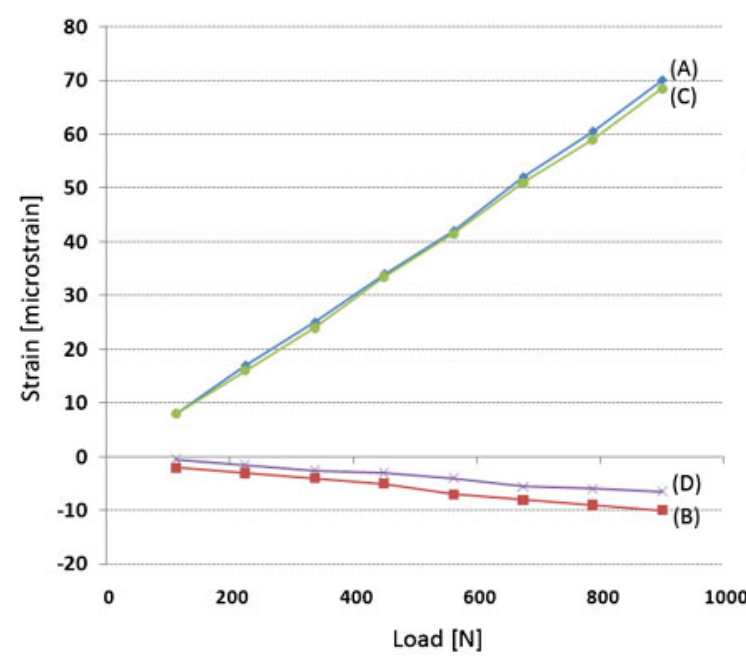

(A) $\rightarrow$-Axial Strain RSG

(B) -- - Transverse Strain RSG

(C) $\rightarrow$-Axial Strain FOS

(D) - Transverse Strain FOS

Fig. 4 Tensile test result comparison

Table 2 Orthotropic material properties

\begin{tabular}{llllllll}
\hline Component & $E_{x}(\mathrm{MPa})$ & $E_{y}(\mathrm{MPa})$ & $E_{z}(\mathrm{MPa})$ & $v_{x y}$ & $v_{x z}=v_{y z}$ & $G_{x y}(\mathrm{MPa})$ & $G_{x z}=G_{y z}(\mathrm{MPa})$ \\
\hline Composite plate & 16900 & 16900 & 3300 & 0.38 & 0.14 & 2900 & 1850 \\
\hline
\end{tabular}



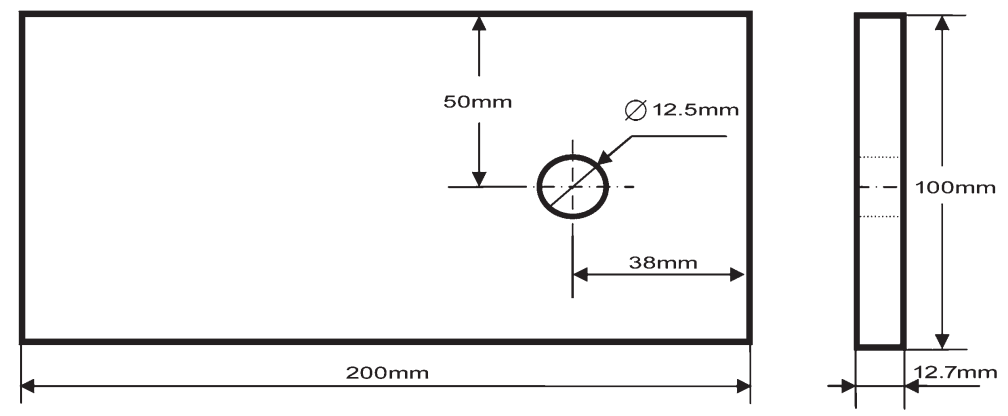

Fig. 5 Composite specimen dimensions and aluminium plate dimensions

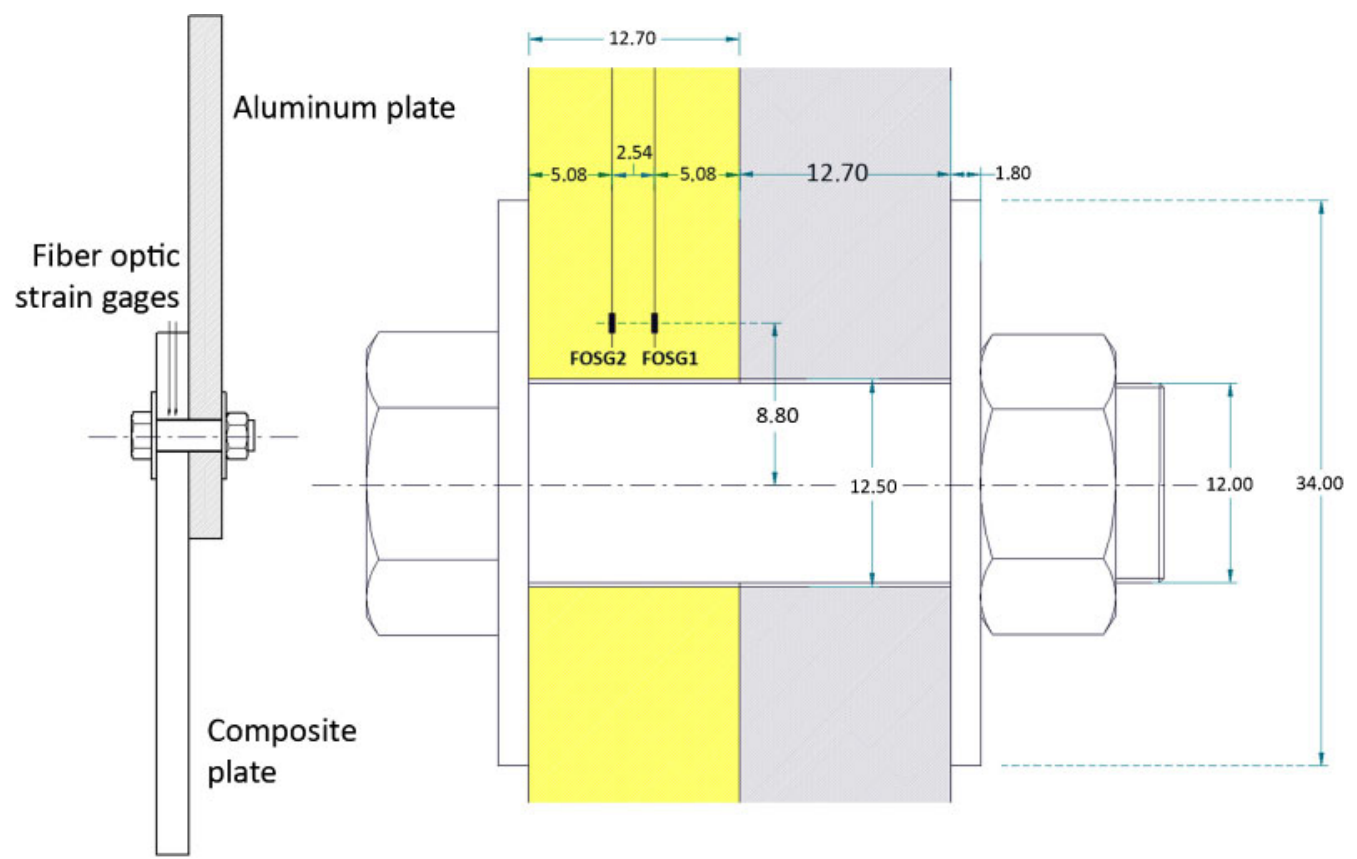

Fig. 6 Positions of fibre-optic sensors

Accurate determination of the clamping load was performed by using Transducer Techniques THC$1 \mathrm{~K}-\mathrm{T} 5000 \mathrm{~N}$ through-the-hole type load cell transducer, as shown in Fig. 7. The transducer is used in combination with a Hewlett-Packard 6200B DC power supply and a Hewlett-Packard 3468A multimeter. The torque on the nut was gradually increased until the preload reached the value of $1200 \mathrm{~N}$. The load cell was left in place for the duration of the experiment.

A small clearance existed between the bolt and the hole (Fig. 6). To be sure that the bolt was centred in the hole and that clearance was evenly distributed around the shank, the preload was always applied before the joint was placed on the loading apparatus. The bolted joint was then subjected to axial load on a loading frame as shown in Fig. 7(a).

The loading procedure consisted of adding dead weights on the load hanger (not shown in the figure).
The loading frame had a mechanical advantage factor of 5. The specimen was loaded at four different load levels according to Table 3.

\section{FINITE-ELEMENT ANALYSIS}

Modern optimization methods for bolted connections are based on FEM because of their high versatility and low cost. This allows the researcher quickly to develop new ideas and validate possible future designs. It is important, however, experimentally to determine the various parameters involved in the process (elastic properties of the elements to be joined, level of preload, etc.) so they can be used as input for the numerical analysis.

Finite-element analysis is a three-step process. First a finite-element model has to be built and load conditions applied. Second, the model is solved 


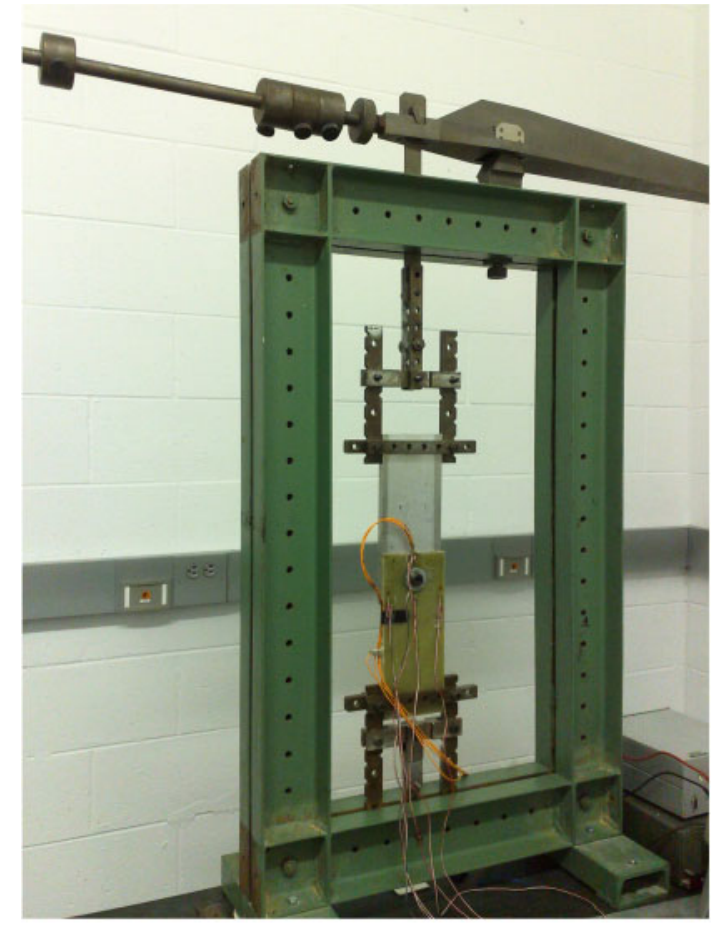

(A)

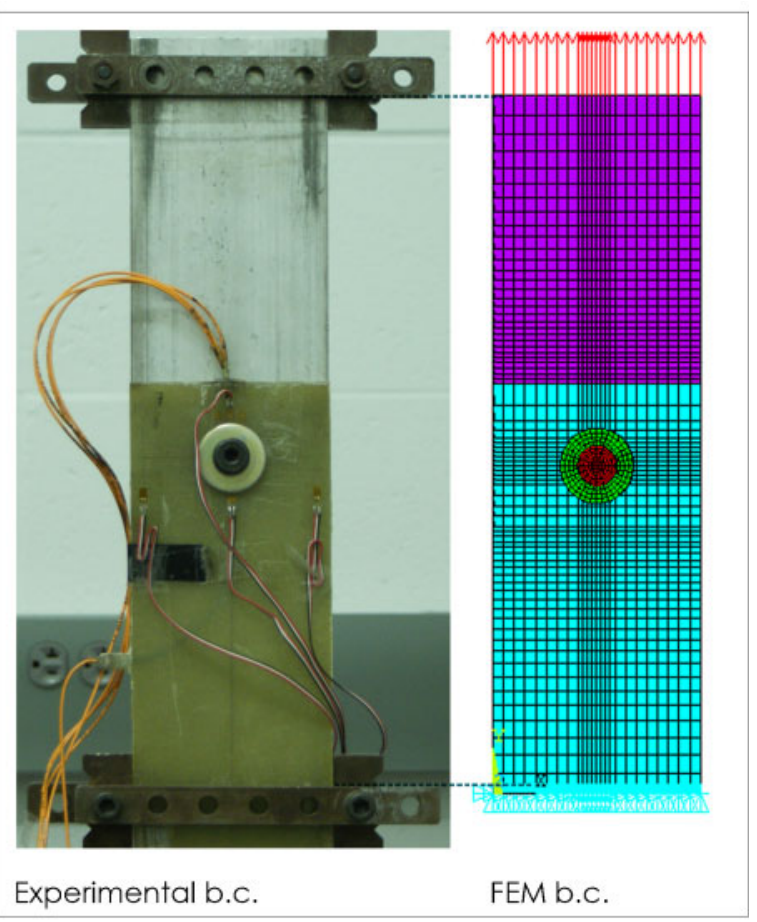

(B)

Fig. 7 (a) Loading frame; (b) boundary conditions

Table 3 Load levels for each test

\begin{tabular}{ll}
\hline Test number & Load on the specimen $(\mathrm{N})$ \\
\hline 1 & 225 \\
2 & 450 \\
3 & 675 \\
4 & 900 \\
\hline
\end{tabular}

using one of the available algorithms. Finally, the third step is the processing and interpretation of the results. The geometry of the model for the present study was obtained through parametric generation of nodes and elements. A piece of code was written in APDL language within Ansys [10]. Parametric

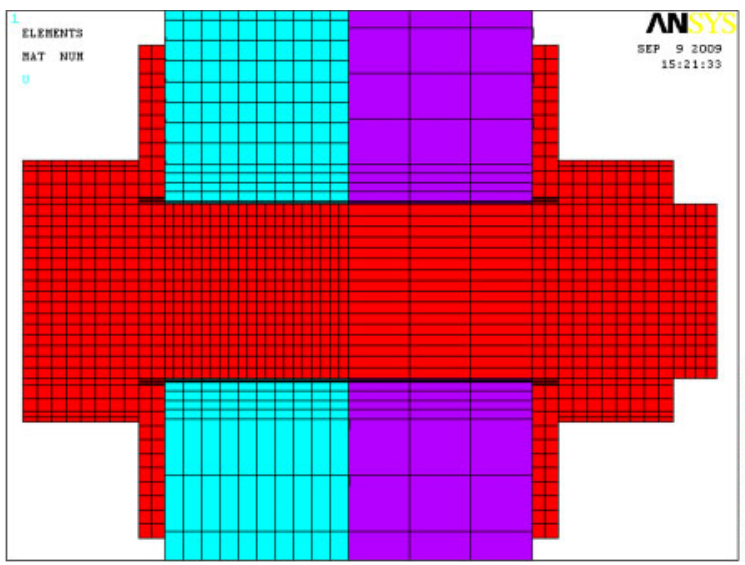

Fig. 8 FEM model discretization: (a) cross-section; (b) assembled joint input values include mesh size around stress concentrations, elastic properties, contacts, loads, and constraints.

The bolted joint was modelled using SOLID164 eight-node elements. Special attention was paid to the meshing process. This step is very important because of the significant impact it has over calculations. A typical mesh is show in Fig. 8.

All the parts of the bolted joint were defined and meshed separately. The Ansys code automatically generates the bolt, the nut, the washers, the composite, and the aluminium plate.

Contact was modelled as node-to-node [11] and a single static coefficient of friction was considered for

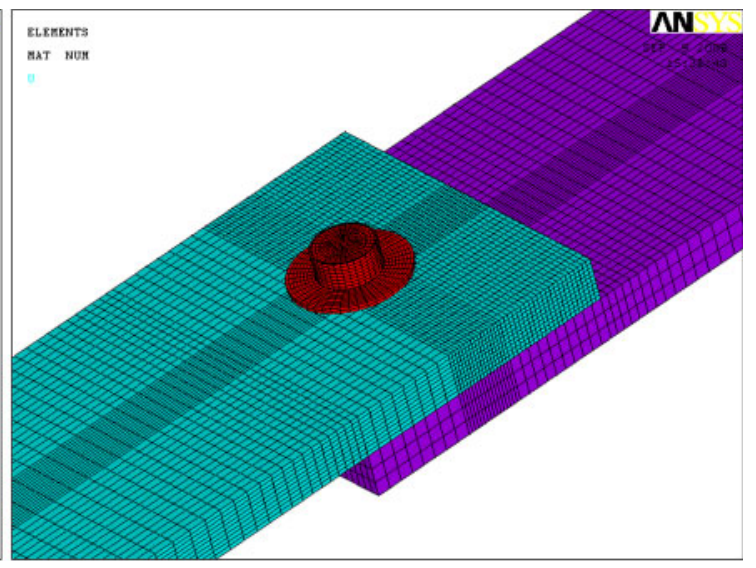


all surfaces in contact. Friction coefficients were assigned separately for each contact pair. The friction coefficient for steel-aluminium contact was set to 0.3 . The friction coefficient for both aluminium and steel with the composite plate was set to 0.4. As a rule of thumb, the stiffer and/or convex surfaces were considered master contact surfaces. For the bolted joint connection model eight contact pairs were modelled using the master-slave principle. The pairs were: bolt to composite plate, bolt to aluminium plate, aluminium plate to composite plate, nut to washer, aluminium plate to washer, bolt to washer, composite to washer, and nut to bolt.

The boundary conditions applied to the finiteelement model simulated in detail the experimental conditions (Fig. 7(b)). The composite plate was fixed at the free end. The load on the bolted joint was applied to the nodes of the top free surface of the aluminium plate. Forces on each node were obtained considering an equal distribution of pressure.

Special care was taken in the simulation of the preloading phase. The goal was to model the load transfer between nut and bolt in a way that closely resembled the actual load exchange between the threads. To achieve this, the total preload was divided by the number $N$ of contact points and the obtained value was assigned to the internal nodes of the nut and to the external nodes of the bolt. Contact elements were introduced only to avoid interpenetrations and to transfer the load; no preload was assigned to these elements.

Figure 9 illustrates the time history for the preload (A and $\mathrm{B}$ ) and for the load on the bolted connection (C through F). Multiple load steps were required to obtain the necessary results to match the experimental loading conditions. The load curve was set so that it was zero until the pretension stage ended. The rate of loading was set to $750 \mathrm{~N} / \mathrm{s}$; this was quite high but closely resembled the actual loading conditions, as dead weights were placed by hand on the loading frame. The rate of loading was the same for all four tests.

The completed model and the loading history were then exported to LS-DYNA. The explicit solver was used to perform the dynamic analysis and to obtain the nodal solutions as a function of time. The finite-element results were offset by the amount recorded at the end of the pretension step, to account for the fibre-optic sensors being reset to zero.

\section{RESULTS}

Experimental results shown in Fig. 10 are an average of multiple experiments. Measurements were repeatable within a few per cent, with a 5 per cent worstcase scenario. It can be observed that the strains are low for all sensors until the load applied to the bolted joint increases over 400 N. For loads exceeding $450 \mathrm{~N}$, an accentuated increase in compression strain gives a clear indication of the jump in the load supported by composite plate.

The results show a reasonably good correlation between finite-element analysis and experiments in the bearing region of the composite plate. The largest difference between experimental and numerical values is 16 per cent.

A study was also conducted to determine the influence of the friction values. Upon further investigation, it was shown that different coefficients of friction do not influence the longitudinal strain values; however, some of the dynamic effects are affected by the coefficients of friction. Because of the presence of clearance between the bolt and the hole, once the load reaches a certain level, the plates start

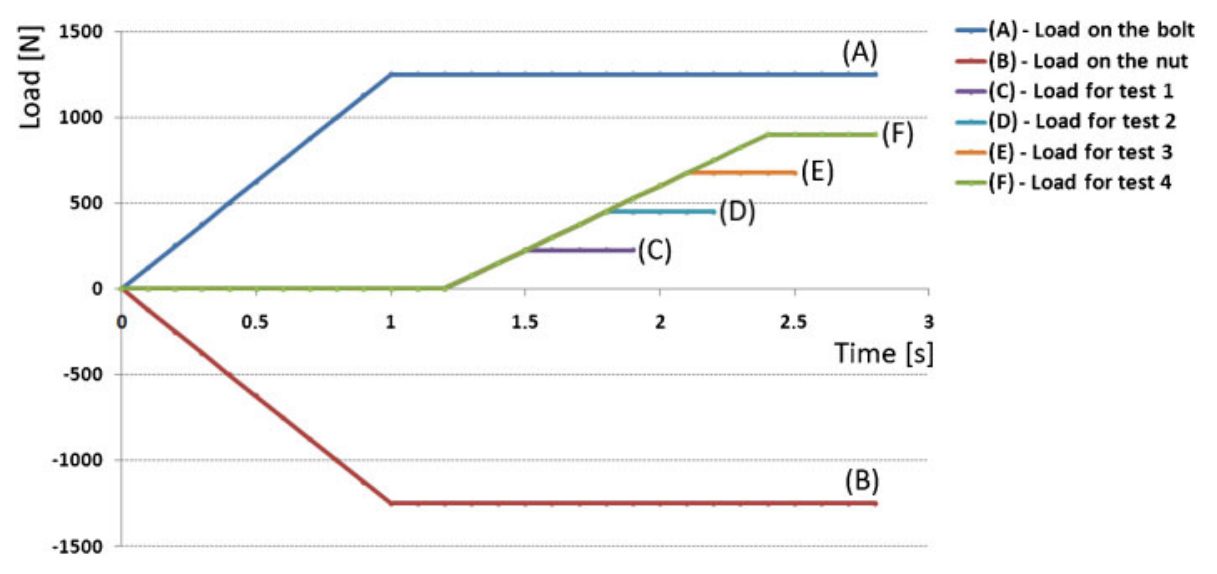

Fig. 9 Time history for the preload and for the load on the joint 


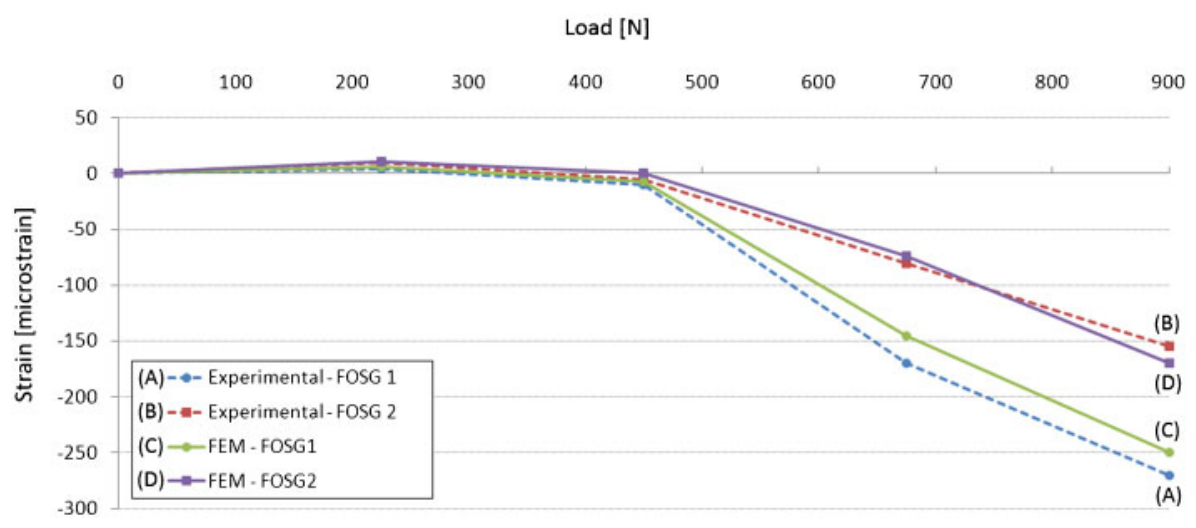

Fig. 10 Numerical and experimental results comparison for the two FOSG locations

to slide with respect to one another. The sliding continues until both plates are in contact with the bolt. The interval of time during which the plates are sliding will be referred to as 'sliding time'. It should be noted that the sliding occurs only for the load levels of tests 3 and 4 (higher loads).

In conclusion, it was observed that the 'sliding time' and the time of first contact between the bolt and edge of the hole are both affected by changes in friction coefficients. Figure 11 shows the start and end time for the sliding, expressed as per cent and referred to the loading time. The values are plotted as a function of the static coefficient of friction $\mu_{\mathrm{s}}$. The ratio between static and dynamic coefficients of friction was $\mu_{\mathrm{s}} / \mu_{\mathrm{d}}=1.33$.

\section{DISCUSSION}

Correct positioning of the optical fibres is extremely important in order to obtain reliable and meaningful

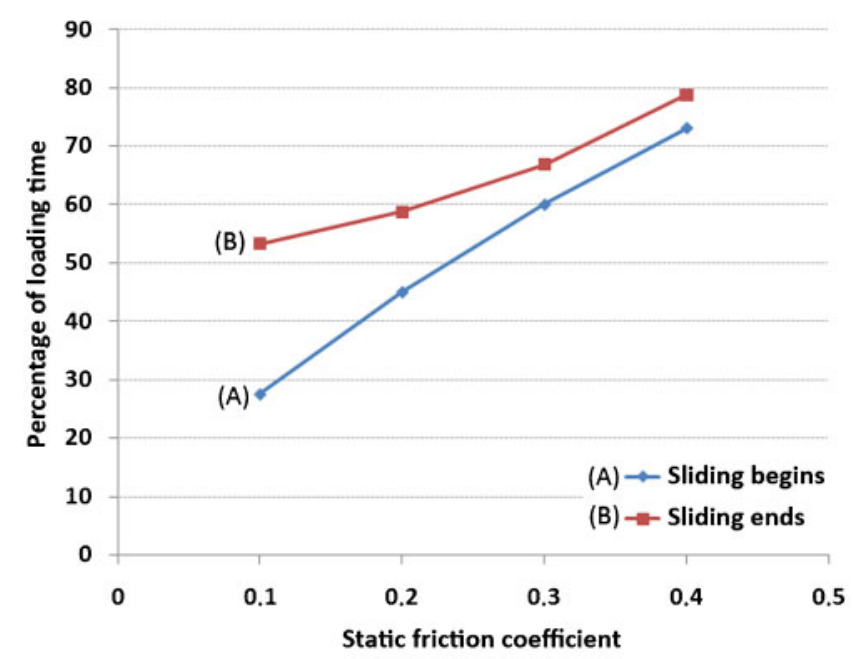

Fig. 11 Influence of the friction coefficient on the sliding time strain values. This is especially true in areas of high strain gradients, such as the edge of the hole. The discrepancy in Fig. 10 was mainly attributed to a small error in the final position of the FOSGs. In order fully to justify this hypothesis, the influence of an incorrect positioning of the gauges was studied.

Figure 12 shows the strain values in the direction of the applied load obtained from the numerical simulations for increasing values of the distance from the edge of the hole. The values are referred to the position of the fibre as originally intended (zero position) and show what values would have been obtained at different locations. The two graphs are obtained for a load of $900 \mathrm{~N}$ and are related to FOSG1 and FOSG2.

Figure 13 shows the per cent error caused by an incorrect positioning of the fibre optics. The values are plotted as a function of the correct position and show (detail A) the range in which the incorrect positioning will result in an error in the strain values equal or less than 16 per cent. From the graph it can be seen that this corresponds to a difference of $0.5 \mathrm{~mm}$ in the strain gauge location.

Once the model is validated against experimental data coming from embedded FOSGs and discrepancies are interpreted and explained, it is necessary to look at the complete 3D solution and analyse the full stress distribution. This provides general trends as well as the location of stress concentrations: this is useful information for the engineering design of similar connections.

Figure 14(a), shows the $\sigma_{\mathrm{y}}$ stress distribution in the composite plate near the hole. The graph is obtained for the last step of the loading procedure.

Line plots were also obtained along curves A, B, and C of Fig. 14(b). Figures 15, 16, and 17 show the $\sigma_{\mathrm{y}}$ contact stress profiles, as a function of the angle $\delta$ shown in Fig. 14(b). For each graph, the strains are plotted for different values of the loading time. 

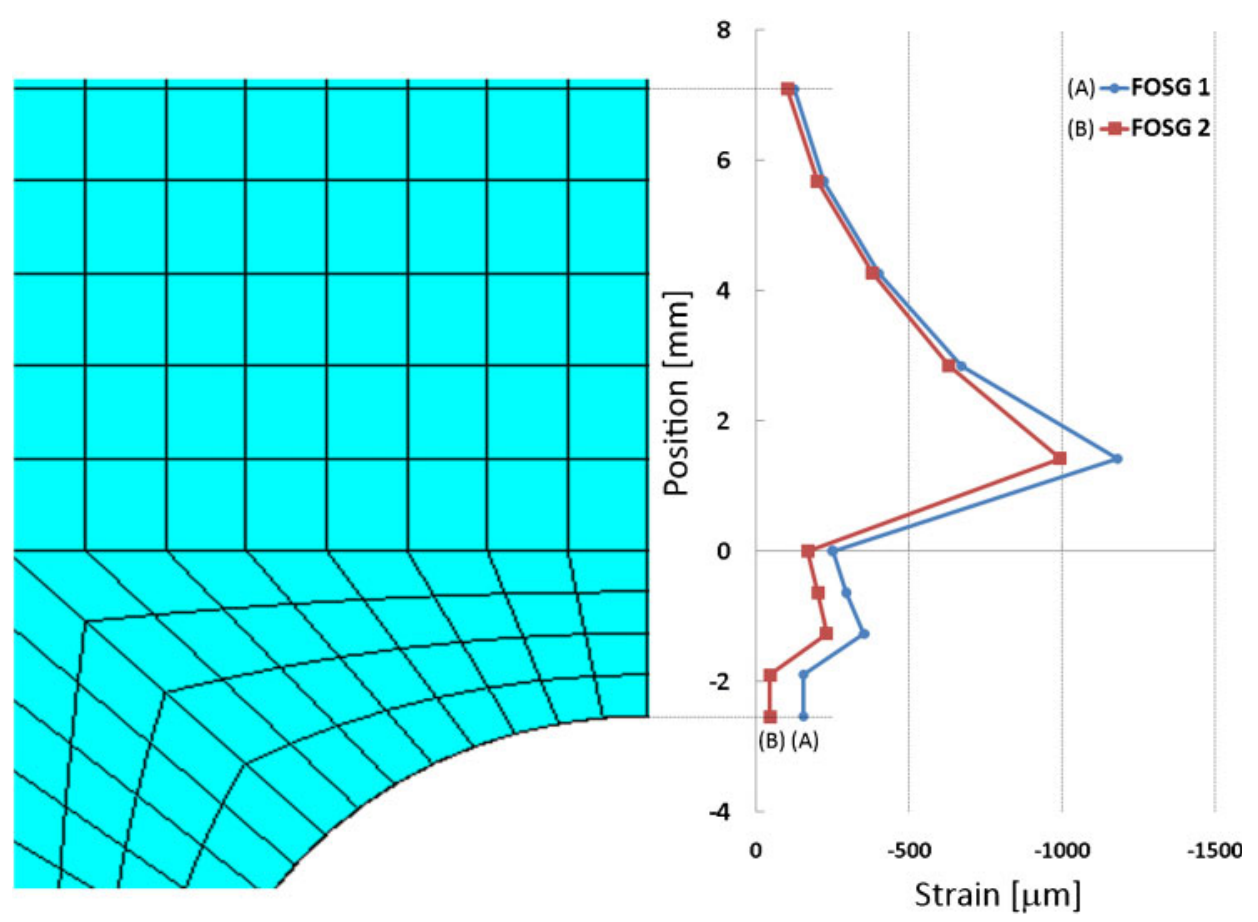

Fig. 12 Strain distribution on the bearing plane. The zero-position is referred to the exact installation of the FOSG
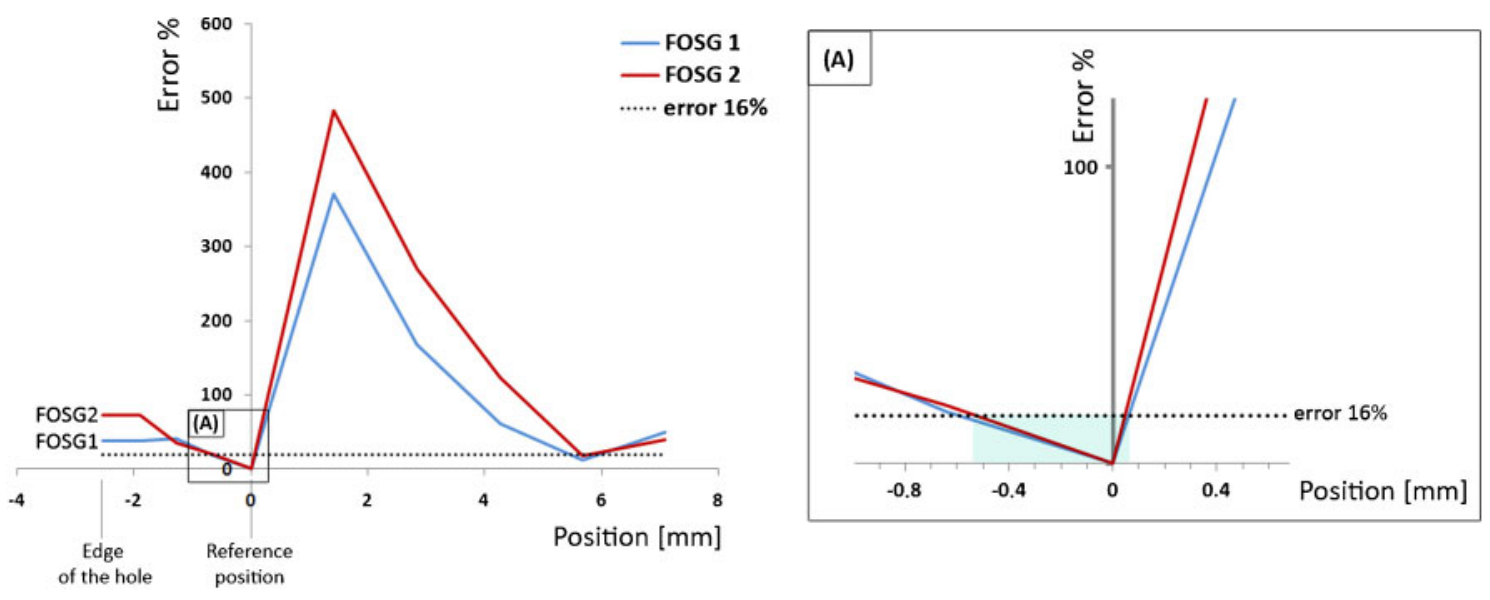

Fig. 13 Per cent error owing to the incorrect positioning of the FOSGs

It can be observed that contact stress values become relevant at 85 per cent loading time. Also, the most stressed area corresponds to point 3 in Fig. 14(a), where the stress value is about twice that of points 1 and 2.

For the optimal design of bolted connections, attention must therefore be focused on the area around point 3 (Fig. 14(a)). The goal is to decrease the stress concentration caused by the bolt-to-hole contact. The extension of contact area is the dominant parameter and it is clearly evidenced in Fig. 17.

Different designs must be evaluated and ranked based on the decrease of stress concentration and on the increase in contact angle. For instance, it is apparent that an increase in bolt-to-hole clearance will induce a decrease in contact area and it is therefore detrimental to the joint resistance. On the other hand, the hole shape might be modified in order to allow for an increase in contact area.

\section{CONCLUSION}

This study was concerned with the evaluation of strain fields in thick composite plates used in singlelap bolted joints. The area of interest was the bearing 


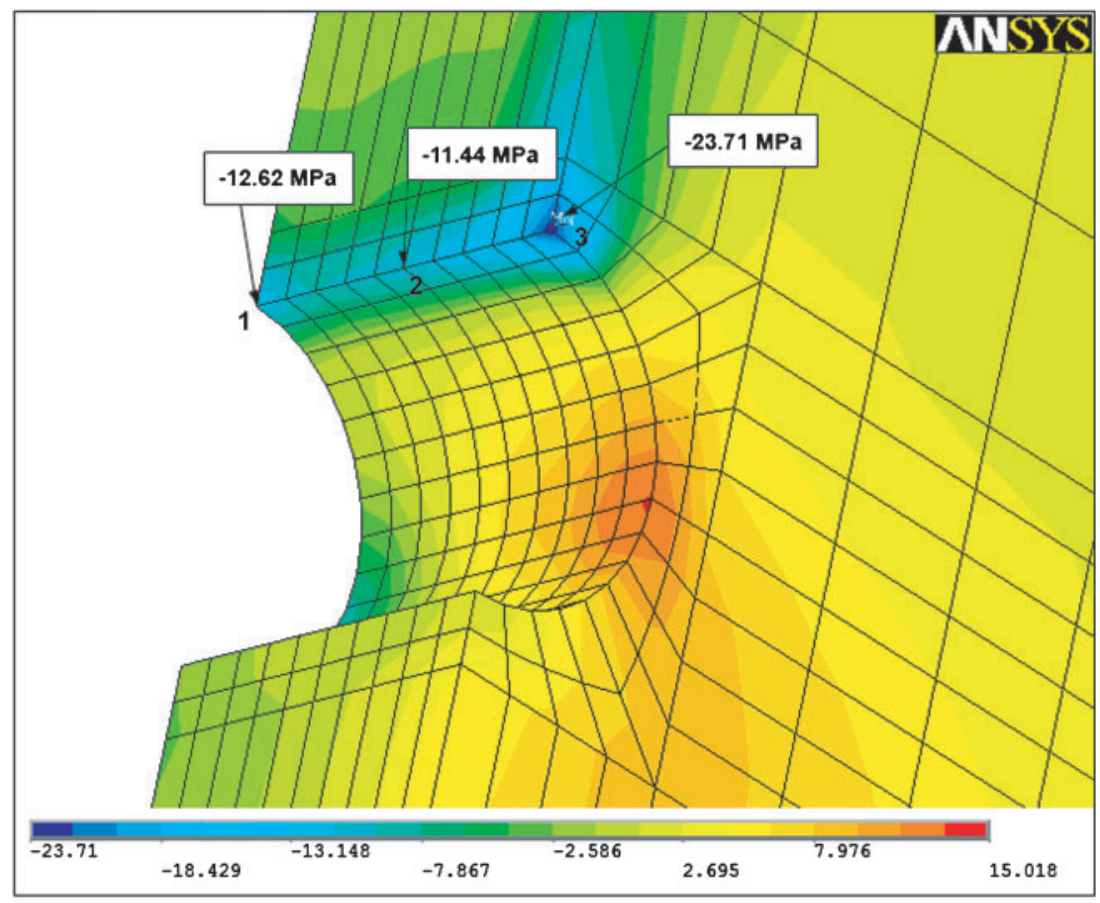

(A)

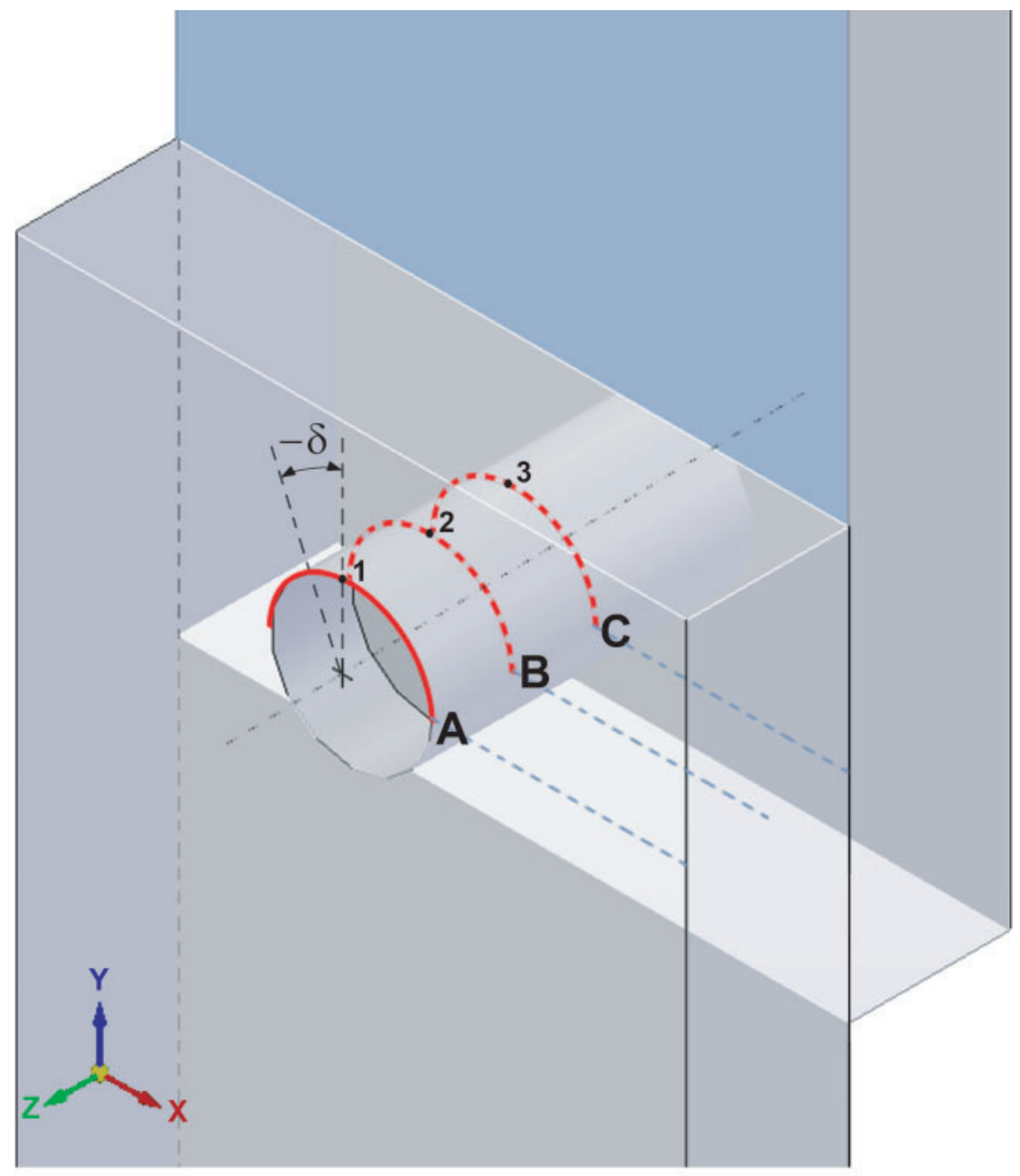

(B)

Fig. 14 (a) Stress distribution in the composite plate near the hole; (b) angle and curve conventions used in contact stress plots 


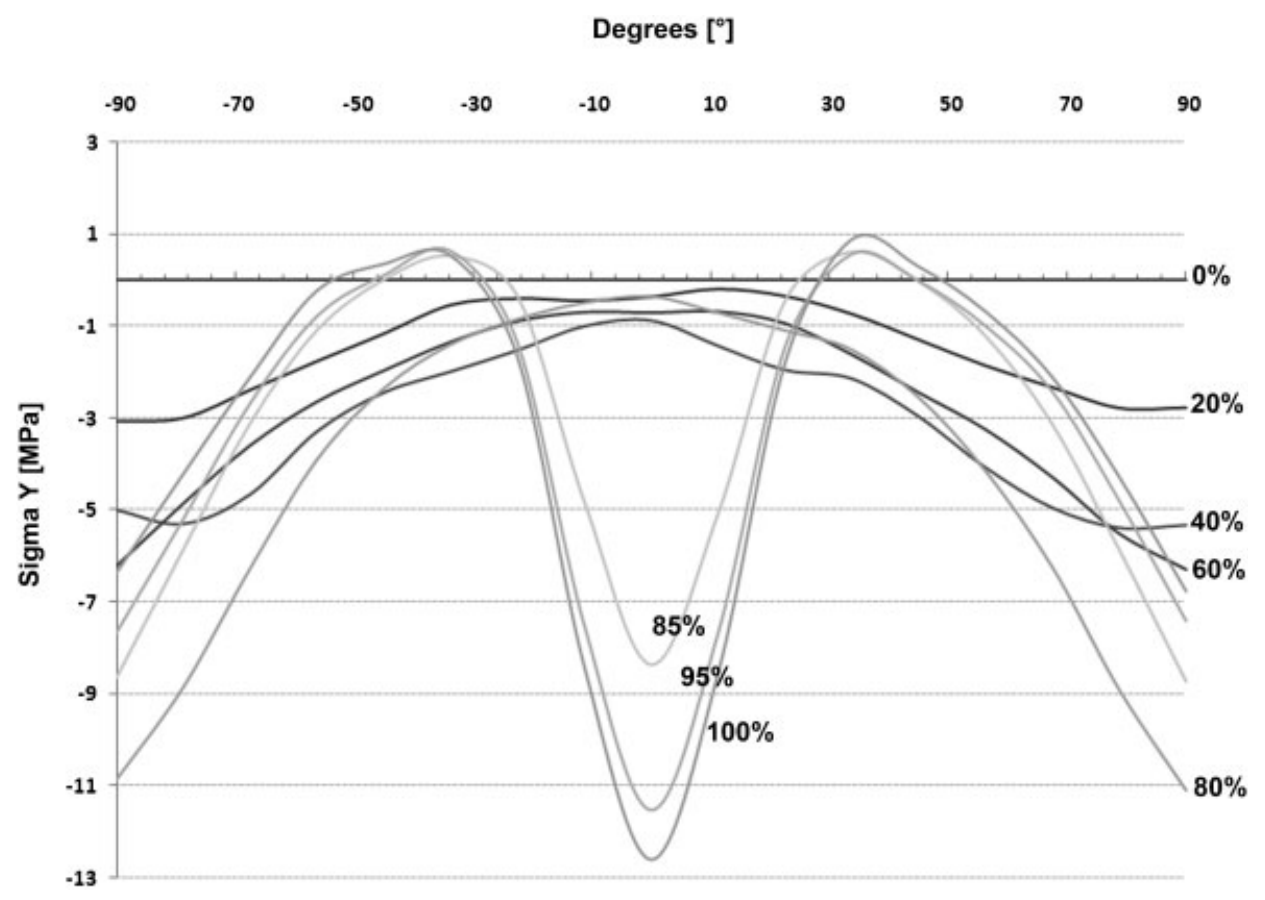

Fig. 15 Contact stress profile along curve A of Fig. 14(b)

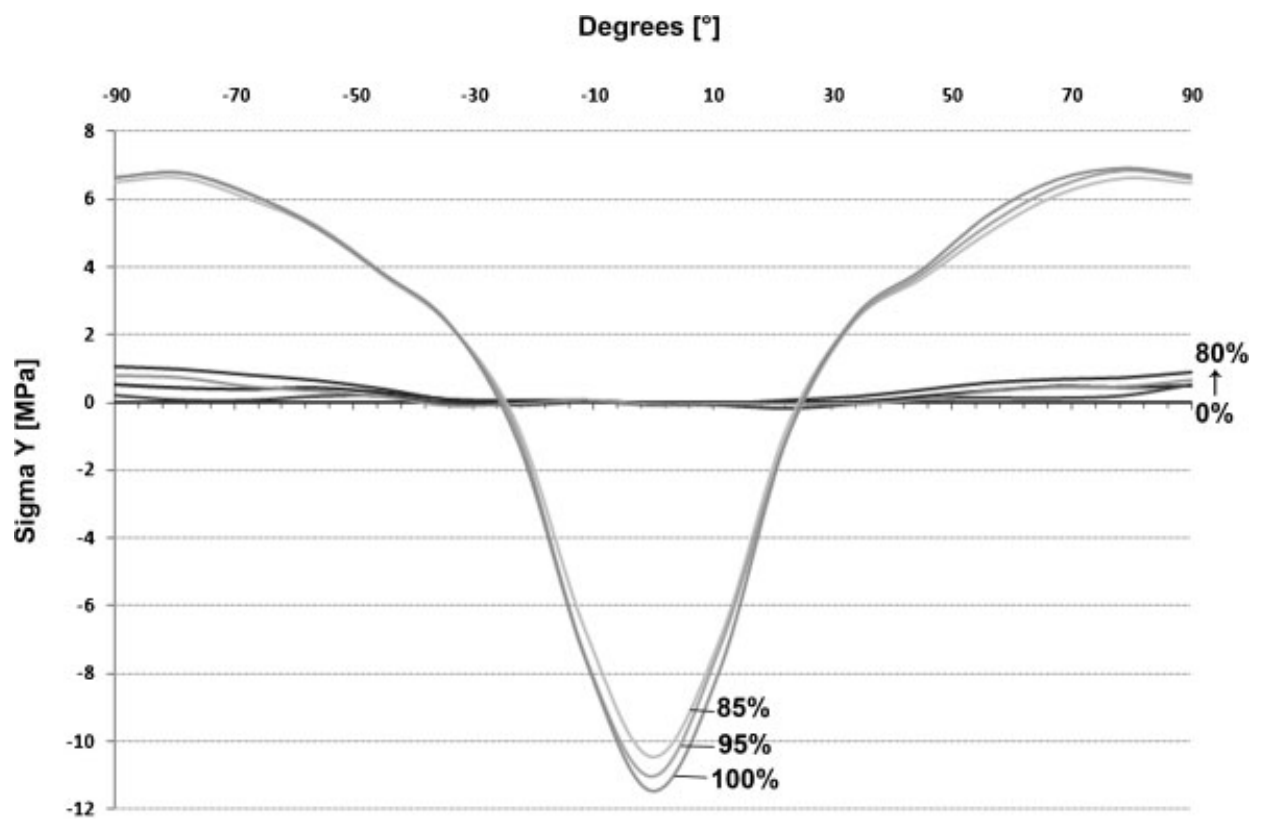

Fig. 16 Contact stress profile along curve B of Fig. 14(b)

plane region close to the bolt hole. The materials used for the two plates forming the bolted joint were aluminium 2024-T4 and E-glass/epoxy. The composite plate had 60 plies and 0.38 volume fraction. Experimental data for the bolted joints were recorded by embedded fibre-optic strain gauges and a through-the-hole type load transducer. Fibre-optic strain gauges were used to obtain internal strains close to the bolt.

The numerical analysis was performed using Ansys as pre-processor and LS-DYNA as solver. Experimentally validated material properties were used for the composite plate. The finite-element analysis correlated reasonably well with the experi- 


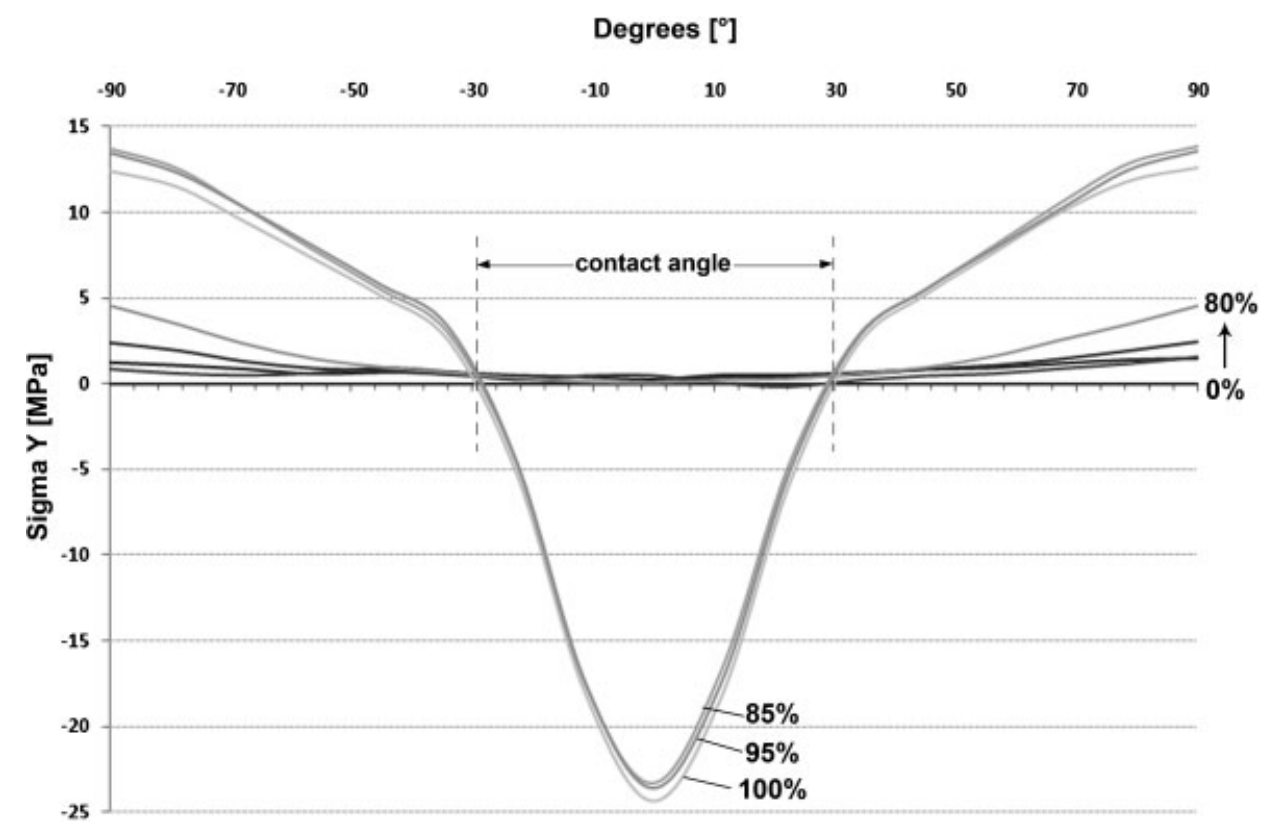

Fig. 17 Contact stress profile along curve C of Fig. 14(b)

ments. The largest difference between the axial strains obtained from the experiments and the finite element was approximately 16 per cent. A study on the influence of incorrect positioning of the FOSG showed that such a discrepancy was probably caused by a small error in the position of the gauges. An additional study was carried out to investigate the effect of the friction coefficients, which ultimately influenced only the dynamic behaviour during the loading phase and had little effect on the final strain values. General design considerations were finally provided, based on the complete 3D finite-element analysis.

(c) Authors 2010

\section{REFERENCES}

1 Ireman, T., Ranvik, T., and Eriksson, I. On damage development in mechanically fastened composite laminates. Composite Structs, 2000, 49, 151-171.

2 Hou, L. and Liu, D. Size effects and thickness constraints in composite joints. J. Composite Mater., 2003, 37(21), 1921-1938.
3 Tong, L. Bearing failure of composite bolted joints with non-uniform bolt-to-washer clearance. Composites Part A: Appl. Sci. Mag., 2000, 31, 609-615.

4 Chen, W., Lee, S., and Yeh, J. Three-dimensional contact stress analysis of a composite laminate with bolted joint. Composite Structs, 1995, 30, 287-297.

5 Ireman, T. Three-dimensional stress analysis of bolted single-lap composite joints. Composite Structs, 1998, 43(3), 195-216.

6 Iancu, F., Ding, X., Cloud, G. L., and Raju, B. B. Three-dimensional investigation of thick single-lap bolted joints. Expl Mechanics, 2006, 45(4), 351-358.

7 Belleville, C. and Duplain, G. White-light interferometric multimode fiber-optic strain sensor. Optics Lett., 1993, 18(1), 78-80.

8 Belleville, C. and Duplain, G. Fabry-Perot optical sensing device for measuring a physical parameter. US patent 5, 392, 117 .

9 FISO. UMI signal conditioner - USER manual (FISO Technologies, Inc., Sainte-Foy, Canada).

10 ANSYS. ANSYS release 11.0 documentation, 2007 (ANSYS Inc., Canonsburg, PA).

11 LSTC Livermore Software Technology Corporation. LS-DYNA keyword user's manual, 2007, Vol. 1, version 971 (LSTC, Livermore, CA). 